\title{
Quantification of Platelet Contractile Movements during Thrombus Formation
}

\author{
Kjersti Tunströmer ${ }^{1}$ Lars Faxälv ${ }^{1} \quad$ Niklas Boknäs ${ }^{1,2}$ \\ ${ }^{1}$ Department of Clinical and Experimental Medicine, Linköping \\ University, Linköping, Sweden \\ ${ }^{2}$ Department of Haematology, Linköping University, Linköping, \\ Sweden \\ ${ }^{3}$ Department of Clinical Chemistry, Linköping University, Linköping, \\ Sweden
}

Thromb Haemost 2018;118:1600-1611.

\author{
Tomas L. Lindahl1,3
}

Address for correspondence Kjersti Tunströmer, MSc, Department of Clinical and Experimental Medicine, Linköping University, 58183 Linköping, Sweden (e-mail: kjersti.tunstromer@liu.se).

\begin{abstract}
Imaging methods based on time-lapse microscopy are important tools for studying the dynamic events that shape thrombus formation upon vascular injury. However, there is a lack of methods to translate the vast amount of visual data generated in such experiments into quantitative variables describing platelet movements that can be subjected to systematic analysis. In this study, we developed experimental and computational protocols allowing for a detailed mathematical analysis of platelet movements within a developing thrombus. We used a flow chamber-based model of thrombosis wherein a collagen strip was used to initiate platelet adhesion and activation. Combining the use of a platelet staining protocol, designed to enable identification of individual platelets, and image processing, we tracked the movements of a large number of individual platelets during thrombus formation and consolidation. These data were then processed to generate aggregate measures describing the heterogeneous movements of platelets in different areas of the thrombus and at different time points. Applying this model and its potential, to a comparative analysis

Keywords

- flow chambers

- thrombosis

- platelet aggregation

- platelet contraction

- fluorescence microscopy on a panel of platelet inhibitors, we found that total platelet intra-thrombus movements are only slightly reduced by blocking the interactions between glycoproteins llb/ $\mathrm{III}$ and $\mathrm{Ib}$ and their ligands or by inhibiting thromboxane synthesis or P2Y12 signalling. In contrast, whereas 30 to $40 \%$ of the platelets movements (for the CD42a-labelled platelets) and $20 \%$ (for the pro-coagulant platelets), within a thrombus, are contractile, i.e., towards the centre of the thrombus, this contractile component is almost totally abolished in the presence of agents inhibiting these pathways.
\end{abstract}

\section{Introduction}

Experimental models of thrombosis give important insights into the events that shape thrombus formation upon vascular injury, and can be used in comparative studies to assess the roles of adhesion molecules ${ }^{1}$ or specific platelet receptors, ${ }^{2}$ to measure the effects of drugs ${ }^{3,4}$ and to quantify the effects of physical conditions such as shear rate $e^{5,6}$ on thrombus formation. In the majority of the available thrombosis models, data from images obtained using time-lapse microscopy are converted into quantitative aggregate variables describing how thrombus volume, surface coverage or platelet accumulation (measured as fluorescence intensity) is changed over time. Such an approach carries the implicit assumption that a thrombus can be conceptualized as a homogeneous entity, the composition of which remain received

March 5, 2018

accepted after revision

June 19, 2018
DOI https://doi.org/

10.1055/s-0038-1668151.

ISSN 0340-6245. 〔c 2018 Georg Thieme Verlag KG
Stuttgart · New York

License terms

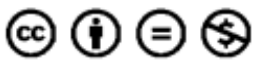


largely unchanged during the course of an experiment. However, as new knowledge highlight the heterogenic and dynamic structure of a developing thrombus, ${ }^{7-10}$ the inability of such models to adequately reflect the highly complex processes that shape thrombus formation have become increasingly clear.

With the build-up of a platelet plug at a site of vascular injury, platelet recruitment occurs in parallel with thrombus densification due to the generation of platelet contractile forces, causing simultaneous compaction and expansion of the thrombus. The contractile movements of platelets within a thrombus start immediately, independently of fibrin formation, ${ }^{11}$ and have important functional implications. ${ }^{12}$ The denser platelet plug may also during later stages affect parameters such as clot elasticity, stiffness ${ }^{13}$ and resistance to fibrinolysis. ${ }^{14,15}$ Apart from lacking information on platelet contraction, thrombosis models based on measurements of thrombus volume tend to systematically underestimate thrombus build-up of platelets and other blood cells, as the volume expansion resulting from platelet recruitment is partially counteracted by the opposing effects of platelet contraction.

Furthermore, it is increasingly evident that not all platelets within a thrombus behave the same; a process of spatial and functional differentiation results in the formation of different intra-thrombus platelet sub-populations with discrete haemostatic activities. ${ }^{16}$ Results from studies performed on in vivo mouse models with mechanical vessel ligation or $\mathrm{FeCl}_{3}$-induced endothelial injury to provoke thrombus formation have identified spatial clustering of pro-aggregatory and pro-coagulant platelets into functionally distinct thrombus microdomains. ${ }^{8}$ Other studies, wherein thrombus formation was studied after laserinduced or micro-puncture injury of the mouse cremaster muscle arterioles, have demonstrated the formation of a stable heterogenic thrombus, characterized by a core of densely packed and highly activated platelets surrounded by a loosely packed shell. ${ }^{7}$ Such a thrombus architecture has important implications for the distribution of coagulation factors and secondary platelet mediators such as thromboxane $\mathrm{A} 2$ and adenosine diphosphate. ${ }^{17}$ These observations strongly suggest that a more complete understanding of thrombosis would require experimental methods capable of mapping the activities and movements of a large number of individual platelets inside the thrombus.

We have previously developed a method for counting the individual platelets in a flow chamber model of thrombosis using time-lapse and Z-stack fluorescence microscopy, ${ }^{18}$ enabling quantification and positional information about the platelets within the thrombus. In this study, we used the acquired information about the position of each platelet to simultaneously track the movements of thousands of platelets during in vitro thrombus formation. This approach enabled a detailed and systematic analysis of how platelet intra-thrombus movements shape the overall thrombus architecture and how these processes are affected by the presence of a panel of clinically relevant platelet inhibitors. The approach was applied to platelets labelled with a plate- let-specific antibody and to pro-coagulant, phosphatidylserine positive ( $\mathrm{PS}+$ ) platelets detected by annexin $\mathrm{V}$, allowing for simultaneous evaluation of the activities of two discrete platelet sub-populations during thrombus formation.

\section{Materials and Methods}

\section{Materials}

Anti-CD42a-AF647 (GRP-P) was from Bio-Rad Antibodies (Oxford, UK), annexin V-AF488 was from Invitrogen Molecular Probes (Carlsbad, California, United States) and the anti-glycoprotein (GP) Ib $\alpha$ antibody (clone 6B4) was a kind gift from professor Hans Deckmyn, University of Leuven, Belgium. ${ }^{19}$

\section{Blood Collection and Fluorescent Labelling}

After informed consent, in accordance with the approval by the regional ethics committee in Linköping (Dnr 2012/38231) and the Declaration of Helsinki, venous whole blood was drawn from healthy volunteers into hirudin sampling tubes (Roche Diagnostics, Mannheim, Germany). Only information about gender and age was linked to the samples, thereby ensuring anonymization of the samples. The blood was used within 4 hours and was stored as recommended by Roest et al. ${ }^{20}$. Annexin V-AF488 (1:100 dilution) was added to the whole blood, and a fraction (5\%) of the blood was incubated with $1 \mu \mathrm{g} / \mathrm{mL}$ anti-CD42a-AF647 antibody. Directly before each flow chamber experiment, the labelled blood fraction was returned to the unlabelled fraction and gently mixed. Inhibitors were added to the whole blood before labelling to ensure that all fractions received equal inhibition. The minimum incubation with inhibitor and label was 30 minutes.

\section{Flow Chamber Construction and Use}

A collagen strip ( $250 \mu \mathrm{m}$ wide) was coated on glass slides with Horm collagen solution $(500 \mu \mathrm{g} / \mathrm{mL}$ ) for 5 minutes (Takeda, Linz, Austria), and a straight polydimethylsiloxane channel (height: $100 \mu \mathrm{m}$, width: $400 \mu \mathrm{m}$ ) was placed perpendicular over the collagen strip. The coating was performed on the day of the experiments. Flow chambers were blocked with bovine serum albumin $(1 \mathrm{mg} / \mathrm{mL})$ for 15 minutes prior to use. The blood was drawn through the flow chamber with a Fusion 200 syringe pump from KR Chemyx (Stafford, Texas, United States). The experiments were run for 20 minutes on a heated stage $\left(37^{\circ} \mathrm{C}\right)$.

\section{Image Capture, Platelet Detection and Tracking}

Z-stack time-lapse images were captured with a wide-field $20 \times$ objective (numerical aperture 0.8 ) on a Zeiss Axio Observer Z1 with a Colibri LED module and a Neo 5.5 scientific CMOS camera (Andor Technology Ltd., UK) controlled by the $\mu$ Manager software (Vale Lab, University of California, San Francisco, California, United States). The Z-stacks, $2 \mu \mathrm{m}$ between slices (total of 27 slices), (both colours) were acquired every 20 seconds, resulting in 60 time frames per experiment. Image processing and platelet detection were performed according to the method described previously. ${ }^{18}$ The method is based on an in-house 
developed Python script that process the acquired images to detect the platelets and determine the $x$-, $y$ - and $z$-position for each individual platelet. Two additional analysis were added, enabling platelet tracking and quantification of the thrombus contraction. Tracking in three-dimensional and time was performed with the Trackpy Python package v0.3.2 from the acquired platelet positions. The detected platelets were tracked between consecutive frames provided the maximum displacement between two frames did not exceed $3 \mu \mathrm{m}$ (set to be the approximate width of a platelet). The displacement vector for each platelet between the time frames was determined along each axis (dvx, dvy and dvz), and as a total length (dv). The contractile component was deduced by projecting each platelet movement vector onto a vector towards the thrombus centreof-mass (median of the platelets $x$ - and $y$-positions, and at the basis of the thrombus along the $z$-axis). A more detailed description is provided in the Supplementary Material (available in the online version).

For the PS+ (pro-coagulant) platelets, which were annexin $\mathrm{V}$ positive (observable as circular shapes), a template-matching algorithm was applied for detection. The template-matching algorithm (from scikit-image) used a disk with increasing size for matching and detection of the platelets. Visualization in graphs, heatmaps, scatterplots and movies have been created using Python modules: NumPy, pandas, matplotlib and Seaborn, and with GraphPad.

\section{Statistics}

Data are presented as mean \pm standard deviation (SD) if not otherwise stated. Statistical comparisons were performed with repeated measures two-way analysis of variance or paired samples $t$-test.

\section{Results}

\section{Quantifying the Effects of Common Platelet Inhibitors on Platelet Accumulation and Thrombus Height During In Vitro Thrombus Formation}

Thrombus formation under flow was induced by collagen, using a custom-made flow chamber setup at a shear rate of $400 \mathrm{~s}^{-1}$. Experiments were conducted with and without the addition of antibodies blocking the interaction between the platelet adhesion proteins (GPIIb/IIIa [abciximab, $10 \mu \mathrm{g} / \mathrm{mL}$ ] and GPIb $\alpha$ [6B4, $8 \mu \mathrm{g} / \mathrm{mL}$ ] and their respective ligands [fibrinogen and von Willebrand factor [vWF]]) or platelet inhibitors either blocking $\mathrm{P}_{2} \mathrm{Y}_{12}$ or thromboxane production (cangrelor $[5 \mu \mathrm{M}$ ] or acetylsalicylic acid [ASA] [25 $\mu \mathrm{M}$ ], respectively).

In the absence of inhibitors, there was a rapid adhesion and aggregation of platelets on the collagen surface ( - Fig. 1 and - Video S1 [showing a time-lapse of the forming thrombus captured during 10 minutes, keep in mind only $5 \%$ of the platelets are visible due to the labelling strategy]).
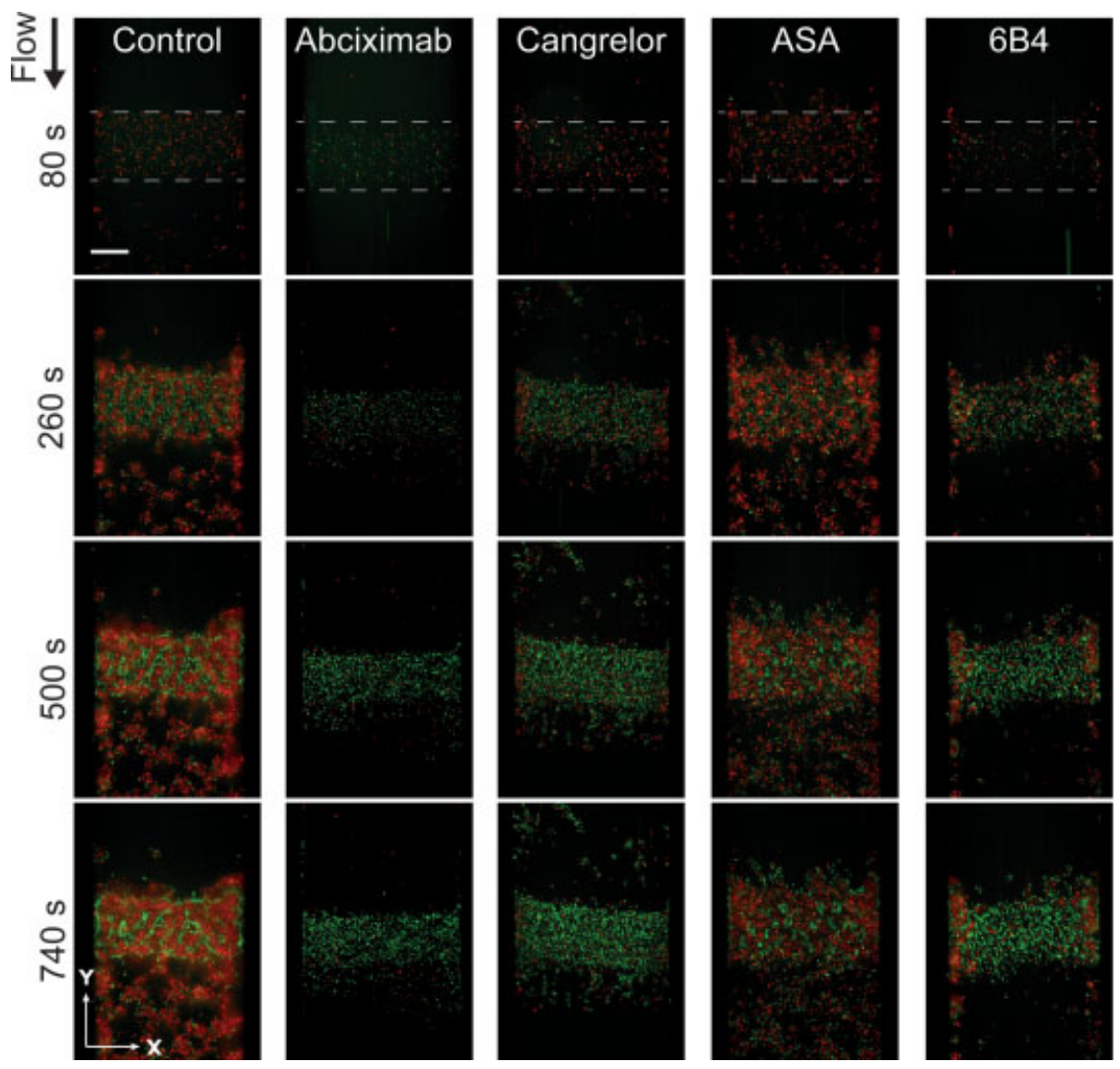
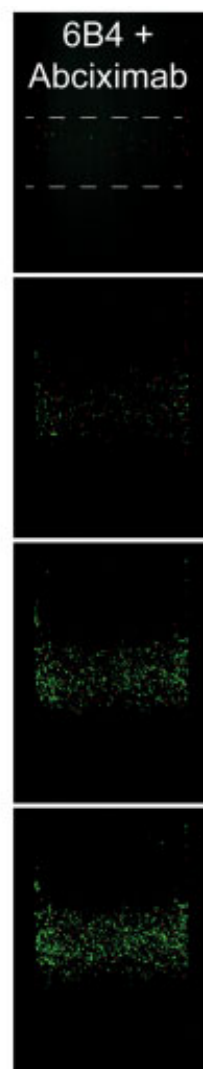

Fig. 1 Thrombus formation on collagen at $400 \mathrm{~s}^{-1}$ with and without the addition of platelet inhibitors. Experiments were performed in a polydimethylsiloxane (PDMS) flow chamber with 5\% CD42a-labelled platelets (red) and annexin V (green), collagen present between the dashed lines. Time-lapse Z-stack images were captured with wide-field fluorescence microscopy. The figure contains a representative set of experiments conducted with blood from a single donor, the images are a two-dimensional (2D) slice acquired near the collagen surface, the experiment was conducted during 1,200 seconds. The flow is directed downwards in the images, and the scale bar represents $50 \mu \mathrm{M}$. 
Measurements of platelet count and thrombus height (-Fig. 2) were performed using a robust and exact method for quantitative analysis as previously described. ${ }^{18}$ The platelet count method, as compared to standard techniques, ${ }^{21}$ enabled reliable and detailed comparative analysis of the effects of the different platelet inhibitors.

The accumulation of CD42a-labelled platelets was significantly decreased in the presence of inhibitors compared to the control ( - Fig. 2 and - Table $\mathbf{1}$ ). The largest decrease was caused by the combination of abciximab and 6B4, with a reduction of thrombus platelet count by approximately $95 \%$, while the smallest but significant difference was seen with ASA. All of the inhibitors produced a dramatic reduction of thrombus height ( - Fig. 2B).

As expected from previous studies, ${ }^{22}$ accumulation of annexin $\mathrm{V}+$ platelets primarily occurred in close contact with the collagen surface ( - Fig. 2B). Interestingly, only ASA and $6 \mathrm{~B} 4+$ abciximab caused a minor but significant effect on the overall accumulation of annexin $\mathrm{V}+$ platelets $(p<0.05$ and $p<0.001$, respectively, over the whole experiment) ( - Fig. 2A). As shown in - Fig. 2B, treatment with ASA resulted in an increased number of $\mathrm{CD} 42 \mathrm{a}$-labelled platelets closest to the collagen-coated surface of the flow chamber, while the number of annexin $\mathrm{V}+$ platelets was decreased, illustrating the importance of thromboxane receptor signalling as a contributor to GPVI-mediated pro-coagulant platelet formation. As shown in - Table 1, the ratio of annexin $\mathrm{V}+$ / total number of platelets in the thrombus was dramatically altered in the presence of the inhibitors, ranging from $0.82 \%$ for the control to $8.59 \%$ for $6 \mathrm{~B} 4+$ abciximab.

\section{Platelet Tracking Enables Detailed Characterization of Platelet Movements within the Thrombus}

The position of all labelled platelets in the thrombus, at each acquired time point, was analysed with Trackpy, a Python toolkit applying the Crocker-Grier algorithm to reconstruct the trajectories of particles from time-lapse images. ${ }^{23}$ This approach enabled large-scale analysis of the movements of individual platelets during the course of an experiment. Platelets were tracked between time frames provided the movement was less than $3 \mu \mathrm{M}$ between two frames. In - Fig. 3, platelet trajectories for the CD42a-labelled and annexin $\mathrm{V}+$ platelets are displayed, with and without $\mathrm{P} \mathrm{Y}_{12}$ inhibition (cangrelor). In the control samples, tracked platelets can be observed to move from surrounding areas towards the centre. This phenomenon was even more evident from a time-lapse visualizing the resulting trajectories ( $\mathbf{- V i d e o} \mathbf{5 2}$ ). The addition of cangrelor reduced the overall movements considerably and the tracked platelets appeared to move in a more stochastic manner. Platelet trajectories for experiments performed with all inhibitors are presented in -Supplementary Fig. $\mathbf{5 2}$ (available in the online version), showing a dramatic shortening of the trajectories for all inhibitors, albeit to a lesser extent with ASA.

To assess whether the relative position of platelets inside the thrombus is a determinant of platelet movements, we plotted the $10 \%$ longest trajectories and the $10 \%$ shortest in - Fig. 4A to visualize their respective position. The longer trajectories appear in the outer parts of the thrombus, while the shorter are more located in the centre of the thrombus.

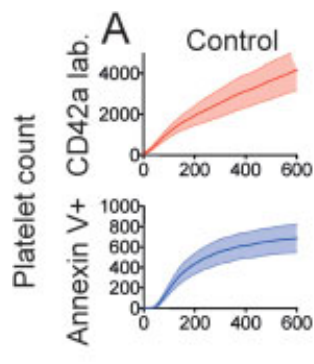

B

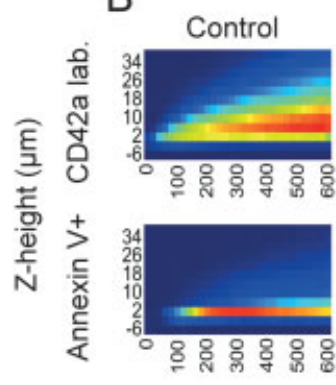

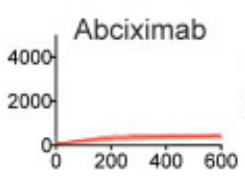
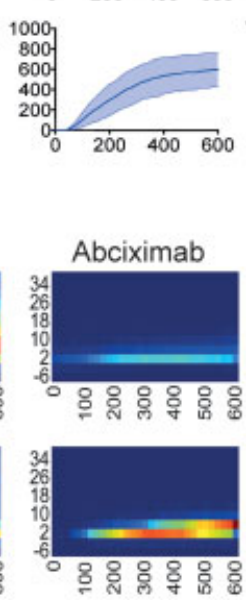

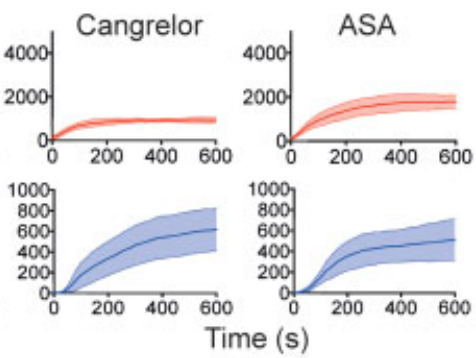

Time (s)
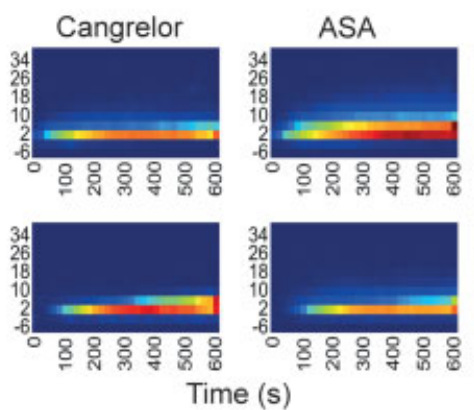
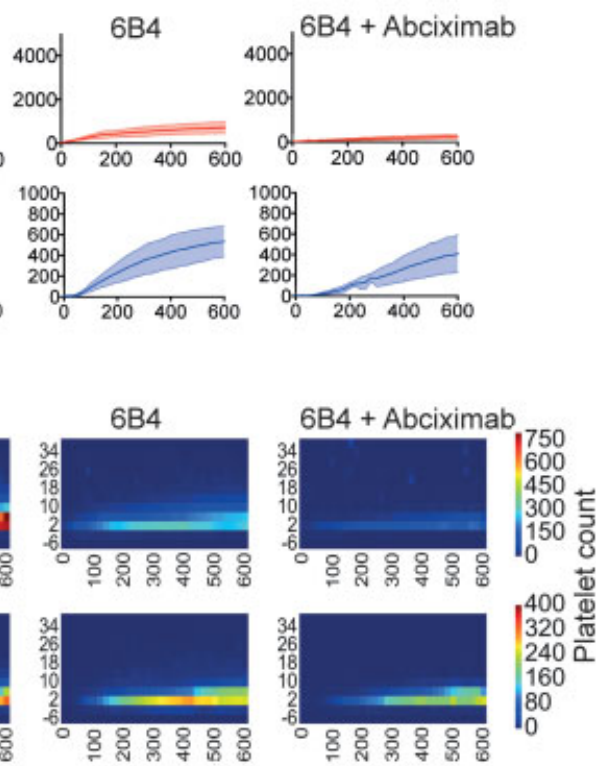

Fig. 2 Platelet count and thrombus height profile. Platelet thrombus formation on collagen was performed in a polydimethylsiloxane (PDMS) flow chamber at a shear rate of $400 \mathrm{~s}^{-1}$ with $5 \%$ CD42a-labelled platelets (red) and annexin V+ labelled platelets (blue). Time-lapse Z-stack images were captured with wide-field fluorescence microscopy and analysed with the platelet count method. ${ }^{18}$ (A) Platelet count over time, mean $\pm 95 \%$ confidence interval $(\mathrm{Cl})(n=6-10)$ for the CD42a-labelled this was $5 \%$ of the total volume of platelets. (B) Mean thrombus height profile over 600 seconds, the colour map indicates the number of platelets in $4 \mu \mathrm{m}$ segments. The plots show the first 600 seconds of the experiments, the total time-lapse (up to 1,200 seconds) is presented in - Supplementary Fig. S1. 
Table 1 Quantification of platelet number and movement

\begin{tabular}{|c|c|c|c|c|c|c|}
\hline & Control & Abciximab & Cangrelor & ASA & 6B4 & $6 B 4+$ abciximab \\
\hline \multicolumn{7}{|l|}{ Mean number (at $600 \mathrm{~s}$ ) } \\
\hline Platelet count - detected (CD42a) & 4,170 & $403^{e}$ & $925^{\mathrm{e}}$ & $1,785^{\mathrm{d}}$ & $722^{d}$ & $239^{\mathrm{e}}$ \\
\hline 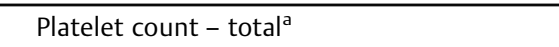 & 83,398 & 8,066 & 18,504 & 35,704 & 14,430 & 4,778 \\
\hline Platelet count - pro-coagulant (annexin $\mathrm{V}+$ ) & 685 & $594^{c}$ & 616 & 509 & 534 & $410^{\mathrm{d}}$ \\
\hline Ratio (pro-coagulant/total) & $0.82 \%$ & $7.36 \%$ & $3.33 \%$ & $1.43 \%$ & $3.70 \%$ & $8.59 \%$ \\
\hline \multicolumn{7}{|l|}{ Mean overall velocity $(\mathrm{nm} / \mathrm{s})$} \\
\hline CD42a & 61 & 52 & 49 & 53 & $40^{\mathrm{e}}$ & $44^{\mathrm{c}}$ \\
\hline Annexin V & 49 & 51 & 38 & 44 & $40^{c}$ & 49 \\
\hline \multicolumn{7}{|l|}{ Mean platelet displacement length $(\mu \mathrm{m})$} \\
\hline CD42a & 6.9 & $2.9^{\mathrm{d}}$ & $3.6^{\mathrm{d}}$ & $3.7^{\mathrm{d}}$ & $4.1^{\mathrm{e}}$ & $3.6^{\mathrm{e}}$ \\
\hline Annexin V & 6.6 & $3.2^{d}$ & $3.3^{\mathrm{d}}$ & $3.8^{\mathrm{d}}$ & $3.8^{d}$ & $3.3^{d}$ \\
\hline \multicolumn{7}{|l|}{ Mean platelet contractile movement $(\mu \mathrm{m})$} \\
\hline CD42a & $2.18^{b}$ & $-0.09^{\mathrm{e}}$ & $0.19^{\mathrm{e}}$ & $0.09^{\mathrm{b}, \mathrm{e}}$ & $0.62^{\mathrm{b}, \mathrm{e}}$ & $-0.09^{\mathrm{e}}$ \\
\hline Annexin V & $2.08^{b}$ & $-0.03^{d}$ & $0.08^{d}$ & $0.17^{d}$ & $0.18^{\mathrm{b}, \mathrm{d}}$ & $-0.08^{\mathrm{e}}$ \\
\hline
\end{tabular}

Abbreviation: ASA, acetylsalicylic acid.

Note: $n=6-10$.

${ }^{\mathrm{a}}$ As $5 \%$ of the platelets were labelled with CD42a, the total platelet count was estimated to be 20 times higher.

${ }^{\mathrm{b}}$ Significantly above zero.

${ }^{c} p \leq 0.05$ for difference in comparison with control.

${ }^{\mathrm{d}} p \leq 0.01$.

$\mathrm{e}_{p} \leq 0.001$.
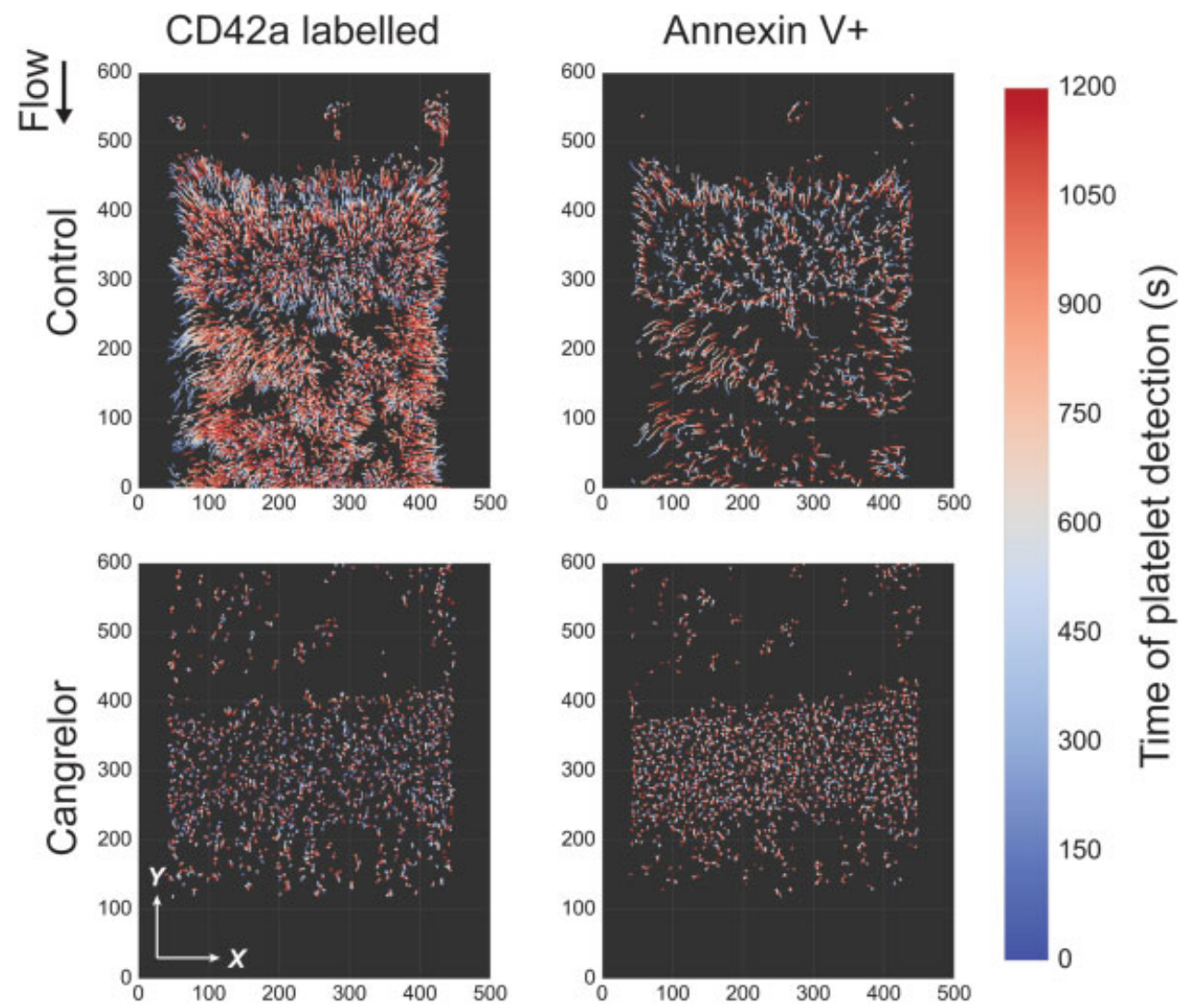

Fig. 3 Platelet movement trajectories obtained from a representative set of time-lapse experiments. Experiments were performed in a polydimethylsiloxane (PDMS) flow chamber at a shear rate of $400 \mathrm{~s}^{-1}$ (without inhibition and with the addition of the adenosine diphosphate [ADP]-receptor inhibitor cangrelor) with 5\% CD42a-labelled platelets and annexin V. Time-lapse Z-stack images were captured with wide-field fluorescence microscopy. In order to make individual tracks more visible, tracks shorter than 5 time frames and $>20 \mu \mathrm{m}$ above the collagen surface were not plotted. Platelet trajectories are given as platelet position in $x$ - and $y$-direction on the representative axis, while the timing is represented by the colour of the trajectory. Experiments were carried out for 1,200 seconds where the time scale turns from blue to red. The axes indicate distance in $\mu \mathrm{m}$. 

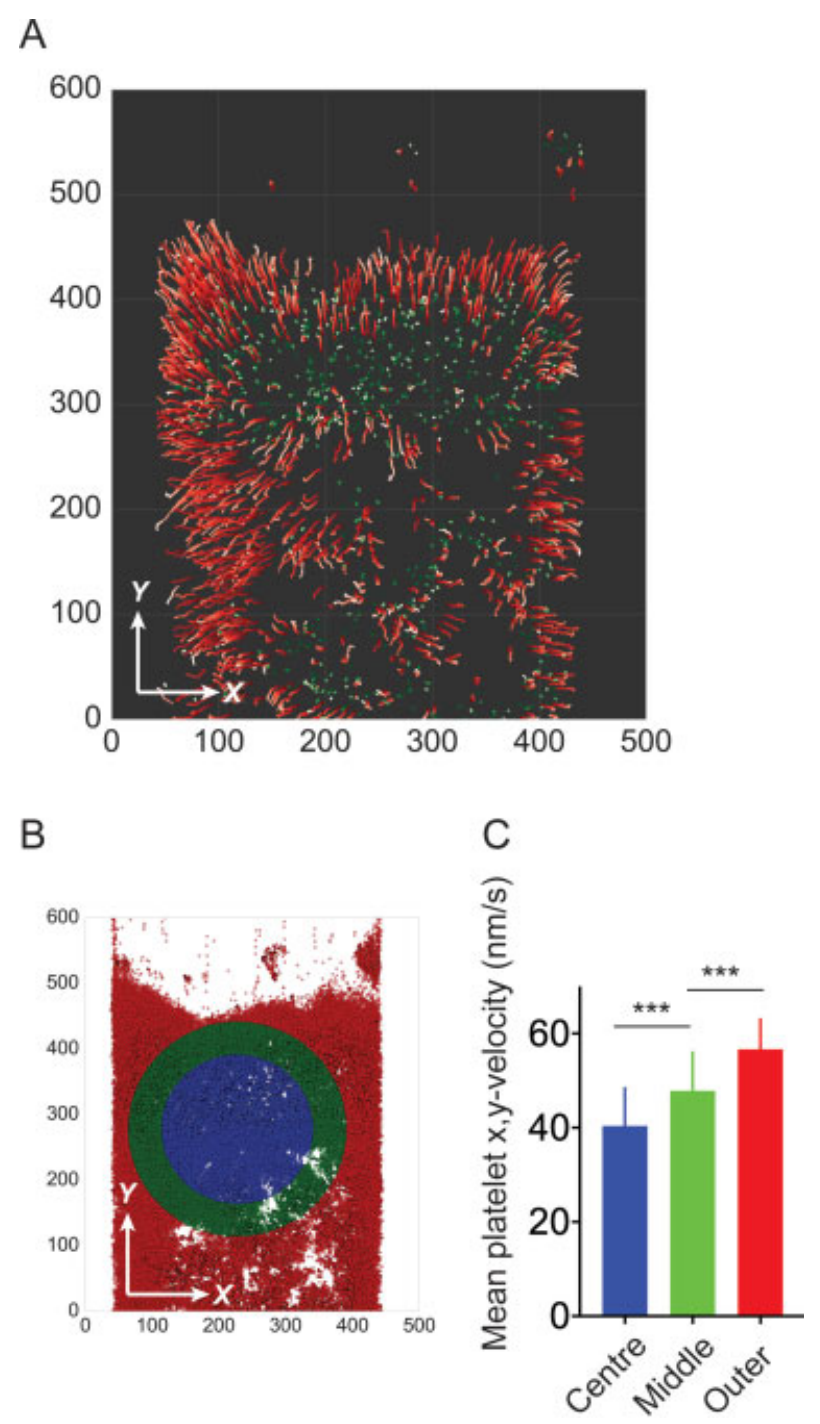

Fig. 4 Platelet movements at different distance from the thrombus centre. (A)The longest (red) and shortest (green) 10\% platelet trajectories, form a representative control experiment where the trajectories were a minimum of 5 time frames long. The axes indicate distance in $\mu \mathrm{m}$. (B) The thrombus formed during a control experiment was divided into three areas defined by the 25 th and 50 th percentile of platelets out from the thrombus centre of mass. The axes indicate distance in $\mu \mathrm{m}$. (C) The mean $x y$-movement was quantified in the three areas described in (B). Values were calculated from time-lapse data of thrombus formation on collagen at $400 \mathrm{~s}^{-1}$ performed in a polydimethylsiloxane (PDMS) flow chamber with $5 \%$ labelled platelets CD42a-labelled platelets. Time-lapse Z-stack images were captured with wide-field fluorescence microscopy. The analysis was performed on the CD42a-labelled platelets. Mean $\pm 95 \%$ confidence interval $(\mathrm{Cl})$, $n=10$.

The platelet movement velocity was quantified at different distances from the thrombus centre of mass ( - Fig. 4B and $\mathbf{C}$ ) and at different heights from the collagen surface (-Supplementary Fig. S3, available in the online version). Our results show a significant increase in velocity for platelets located around the edges of the thrombus structure in comparison with platelets positioned in the thrombus core. In addition, the trajectories of platelets located in dense clusters were generally longer than those of solitary platelets, a phenomenon maybe caused by platelet-platelet crosstalk via GP receptors and paracrine signalling and/or due to the increased mechanical forces in the dense clusters.

Individual platelet movements along the $x$-, $y$ - and $z$-axis, were quantified and illustrated ( $\mathbf{- F i g}$. 5A) by a colour map indicating the platelet velocity (data analysed at $600 \mathrm{sec}-$ onds). As with previous figure, the platelet movements mainly took place on the outer edges of the thrombus, with movement along the $x$ - and $y$-axis directed towards the middle of the thrombus. Movements directed along the $z$ axis of the thrombus occurred mostly at the edges and were more frequently directed upwards from the collagen-coated surface in the base of the flow chamber.

To provide a quantitative measure of the distribution of platelet movements geometrically during the course of the experiments, we calculated the net movements for every tracked platelet along each axis (-Fig. 5B). To facilitate statistical analysis, experiments were divided into four time intervals of which mean and SD were calculated. The movements of the two platelet sub-groups were analysed separately. Along the $x$-axis the movement was symmetrical towards the centre, resulting in a mean net movement close to zero for all conditions throughout the experiments. The movement along the axis of flow, $y$-axis, was affected differently by platelet inhibition. The CD42a-labelled platelets initially moved in an upstream direction during thrombus development ( $13 \pm 5 \mathrm{~nm} / \mathrm{s}$ for the control; mean $\pm \mathrm{SD}$ ). This initial movement was significantly reduced by the addition of abciximab, cangrelor and 6B4 + abciximab $(p<0.01)$. Treatment with abciximab caused the annexin $\mathrm{V}+$ platelets to move downstream, supposedly due to a combination of increased exposure to drag forces caused by the slower accumulation of platelet and an attenuation of the attachment of platelets to the surface of the flow chamber due to the inhibition of GpIla/IIIa-fibrinogen binding (as fibrinogen may be part of the collagen surface after blood contact). Along the $z$-axis, the initial net movement of the CD42a-labelled platelets was downwards towards the collagen surface ( $-9 \pm 6 \mathrm{~nm} / \mathrm{s}$ for the control [0-199 seconds]). This initial movement was significantly reduced by treatment with abciximab, alone or in combination with 6B4 $(p<0.05)$. In contrast, the annexin $\mathrm{V}+$ platelets exhibited a small but significant movement upwards from the collagen surface towards the outer layers of the thrombus. This tendency was amplified by treatment with abciximab or cangrelor but counteracted by treatment with ASA. All movements along the $z$-axis decreased during the later stages of thrombus formation.

From visual inspection, the movements of platelets in the control experiments (-Figs. 3 and 5) appeared to be coordinated. To test this hypothesis, a support vector machine regression approach was used (Python scikit-learn) to estimate the general movement in different regions of the thrombus ( $x$ - and $y$-axis movements). The CD42a-labelled platelets initially contracted into smaller aggregates (as seen in the 0 - to 199-second image), which with time conjoined into larger aggregates (during the 200- to 399-second) that 

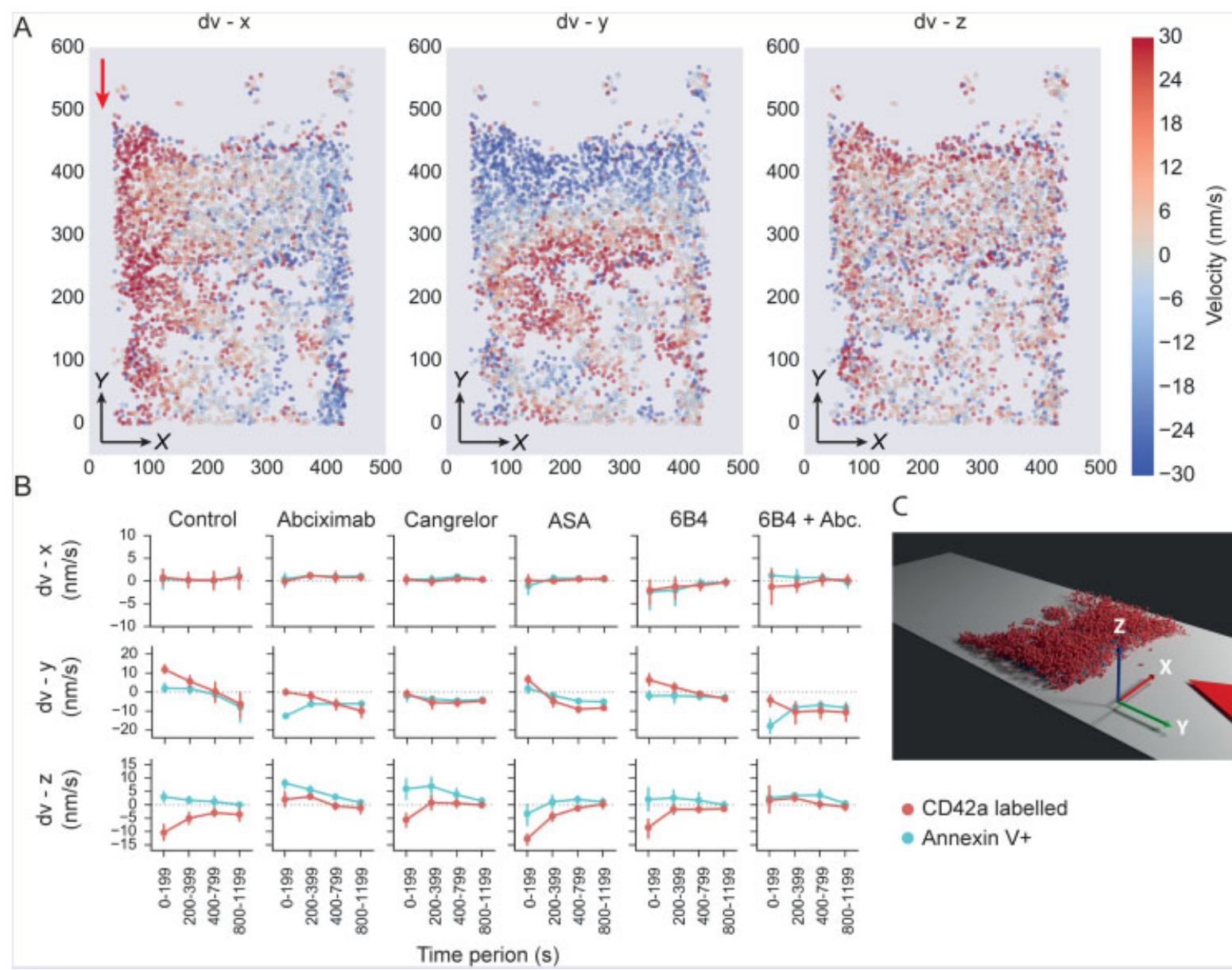

C

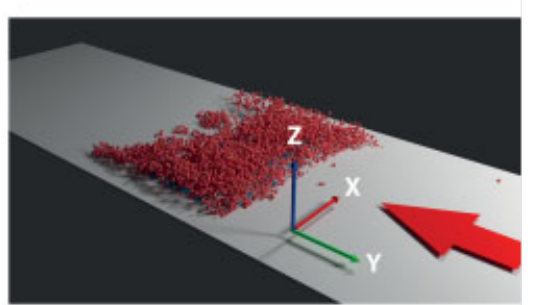

- CD42a labelled

- Annexin V+

Fig. 5 Platelet movement along each axis. The movements of individual platelets were calculated from time-lapse data of thrombus formation on collagen at $400 \mathrm{~s}^{-1}$ with and without the addition of platelet inhibitors. Experiments were performed in a polydimethylsiloxane (PDMS) flow chamber with 5\% CD42a-labelled platelets and annexin V+ platelets. Time-lapse Z-stack images were captured with wide-field fluorescence microscopy. (A) The velocity of individual platelets is indicated by the colour scheme, on a thrombus viewed from above. Red indicates the speed of the movement in direction along the axis and blue indicates the velocity in the opposing direction. Data from movements in a representative experiment at 600 seconds, each dot represents 20 platelets (as on $5 \%$ of the total number of platelets were labelled). The axes indicate distance in $\mu \mathrm{m}$. (B) The mean movement $(\mu \mathrm{m} / \mathrm{s})$ over 4 time-stages along the $x$-, $y$ - and $z$-axis, CD42a-labelled platelets (red) and annexin $V+$ platelets (blue). Mean $\pm 95 \%$ boot strapped $\mathrm{Cl}(n=6-10)$. (C) Directions of the axes in relation to the blood flow.

finally participated in a more homogeneous movement towards the centre of the thrombi (from 400 seconds) as shown in -Fig. 6.

\section{Measuring Thrombus Contraction by Quantification of the Contractile Movements of a Large Set of Individual Platelets}

To obtain an accurate quantitative measure of clot contraction, the contractile component of the platelet movement vector $\left(v_{x y z}\right)$ was determined for each tracked platelet in each time frame. The contractile component was calculated by projecting $\mathrm{v}_{\mathrm{xyz}}$ on to a vector directed towards the thrombus centre of mass $\left(v_{\text {cent }}\right)$ ( - Fig. 7A). The mean overall movement velocity (mean over the individual platelets within each time interval, independent of direction), mean length of the platelet displacement (vector from the platelet starting position to the final position, within each time interval) and the mean contractile component (shown as a percentage of the overall movement) was determined for all platelets in the formed thrombus with and without treatment of platelet inhibitors (-Fig. 7B-E for control, abciximab and cangrelor, and for all inhibitors - Supplementary Fig. S4, available in the online version).

As shown in -Fig. 7D, the contractile component amounted to a considerable fraction of the overall movement ( - Fig. 7B) in the control experiment and increased during the first three phases (0-799 seconds) of thrombus formation with a maximum value of $38.3 \pm 7.4 \%$ for the CD42alabelled platelets and $25.4 \pm 10.3 \%$ for the annexin $V_{+}$ platelets. Both the net overall movement and the contractile component was significantly lower among the annexin $\mathrm{V}+$ platelets in comparison with the CD42a-labelled platelets during the course of the experiments $(p<0.001$ at 0 799 seconds for both, $p<0.01$ at $800-1,199$ for the overall velocity and $p<0.05$ at $800-1,199$ for the contractile component), while the displacement length was identical between the two platelet sub-populations during the three later phases of the experiments. Interestingly, during the last 

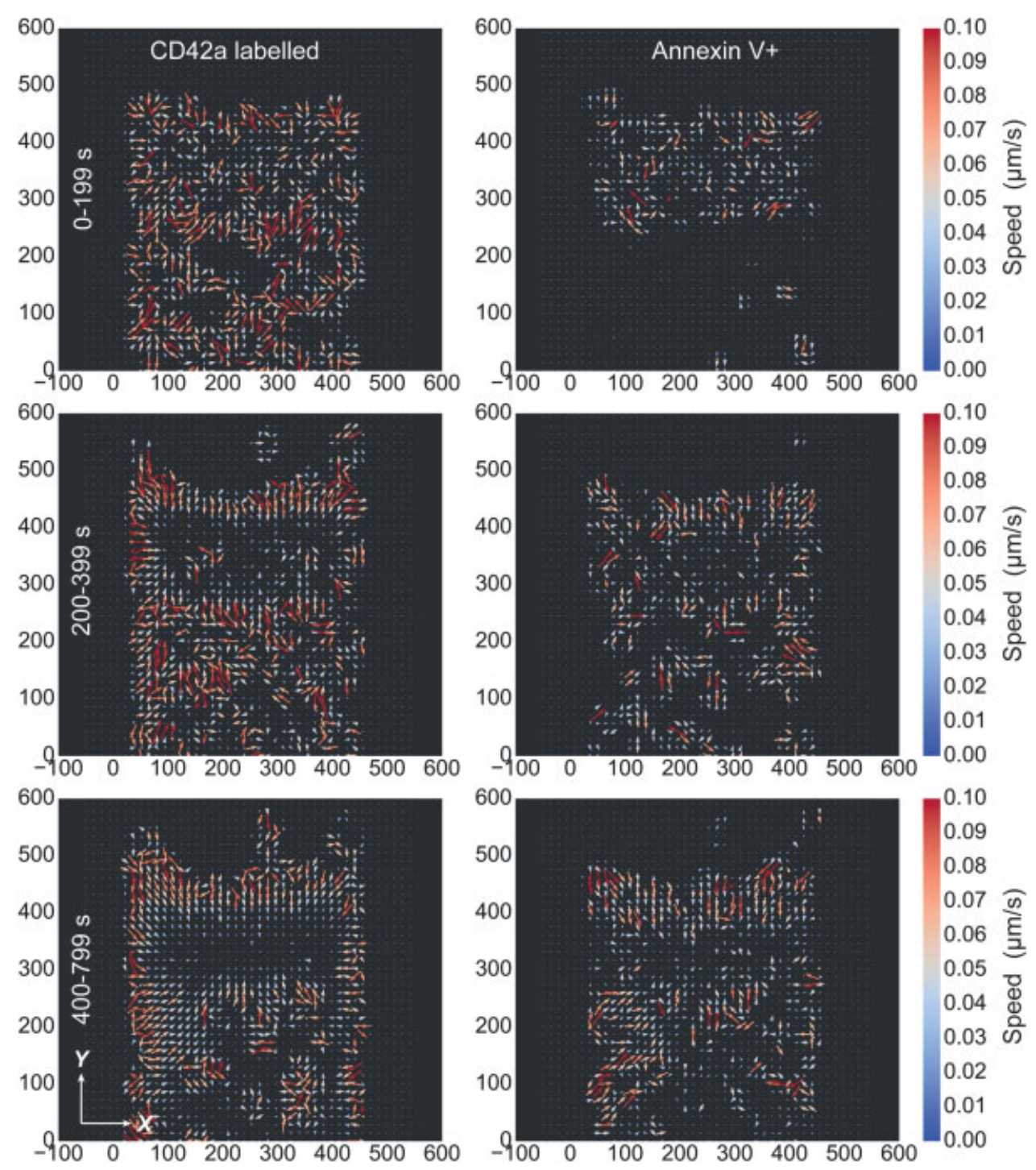

Fig. 6 Regression analysis of movements during thrombus formation. The regression analysis (support vector machine regression) determines the direction and size of the overall movement in all areas of the thrombus. Thrombus formation was induced by collagen at $400 \mathrm{~s}^{-1}$, in a polydimethylsiloxane (PDMS) flow chamber with 5\% CD42a-labelled platelets and annexin V+ platelets. Images were captured with wide-field fluorescence microscopy. One representative experiment is shown. The length and colour of the arrows indicate platelet velocity as described by the colour bar. The axes indicate the distance in $\mu \mathrm{m}$.

phase of the experiments (800-1,199 seconds), the contractile movements of the CD42a-labelled platelets reached a plateau and even decreased slightly, while the contractile movements of the annexin $\mathrm{V}+$ platelets continued to increase, resulting in a less pronounced relative difference between the two platelet sub-populations. The overall velocity at 600 seconds, the total displacement length and contractile movement over the entire experiment (20 minutes) are also presented in - Table $\mathbf{1}$.

Treatment with inhibitors resulted in a pronounced inhibition of the contractile component and reduction of the mean length of the platelet displacement, while having only a minor effect on the mean overall movements. There was some remaining contractile component in the sample inhibited by 6B4, supposedly due to the inhibitors' inability to block platelet aggregation near the flow chamber walls (-Fig. 7E and - Table 1).
Irrespective of which platelet inhibitor was used, the mean overall movement of the CD42a-labelled platelets was initially reduced (0-199 seconds), compared to the control $(p<0.05)$. With the addition of abciximab, the annexin $\mathrm{V}+$ platelets initially moved significantly more than those labelled for CD42a, supposedly due to increased exposure to drag forces, a similar pattern as in - Fig. 5 . However, in comparison to the control, the inhibitors had no general effect on the overall movement of the annexin $\mathrm{V}+$ platelets.

The net contractile movement was determined at different distances from the collagen-coated surface along the $z$ axis ( - Fig. 7E) and was found to increase dramatically higher up in the thrombus structure, with significant differences in contraction between the three lowest sections $(p<0.001)$. The addition of inhibitors strongly attenuated the contractile component, and it was only the 4 to $16 \mu \mathrm{m}$ section with 
A

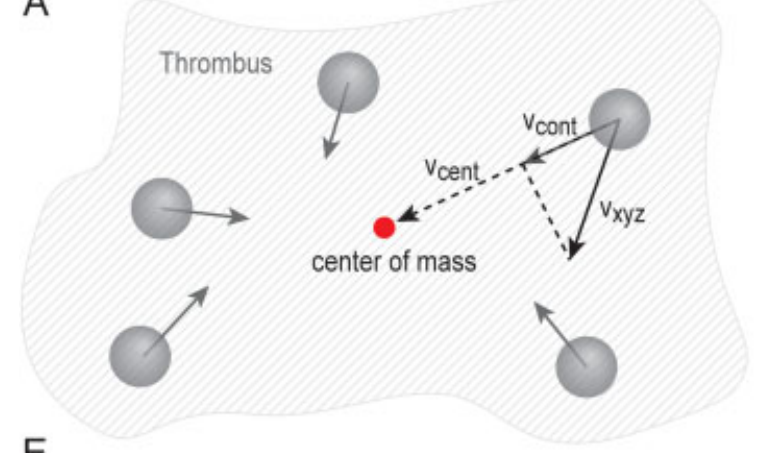

E

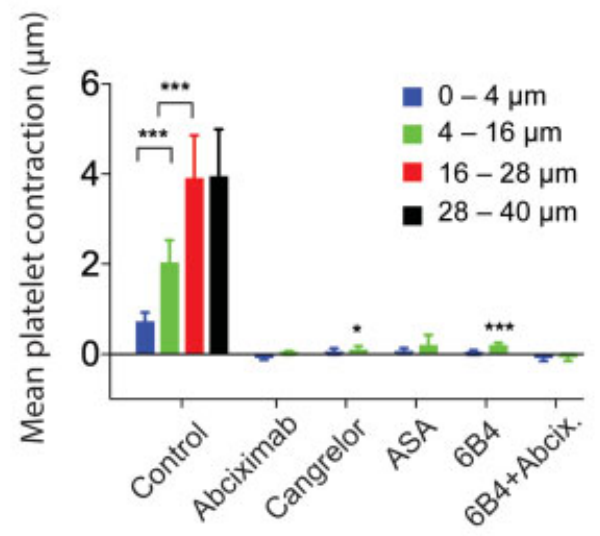

B

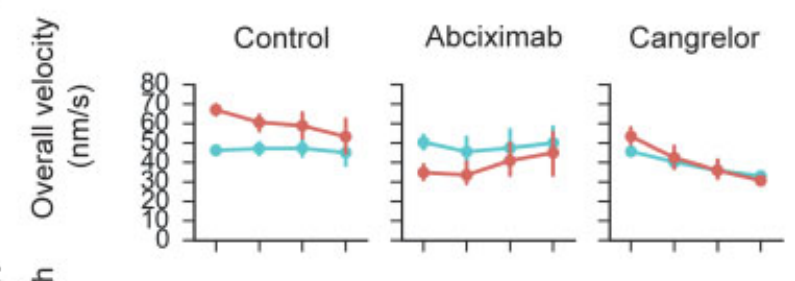

C

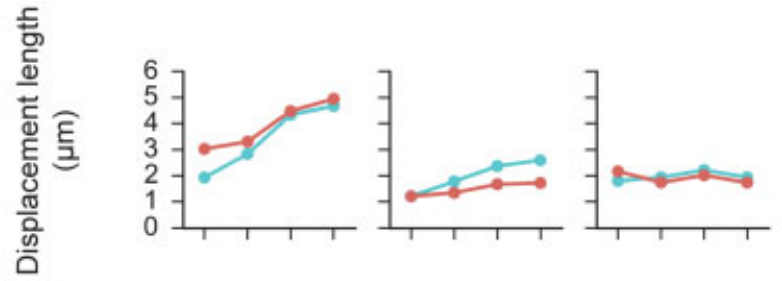

D

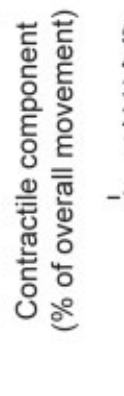

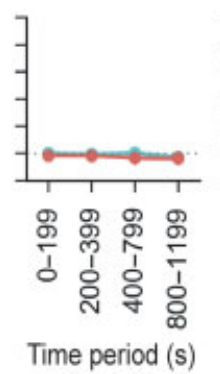

- Annexin $\mathrm{V}^{+}$

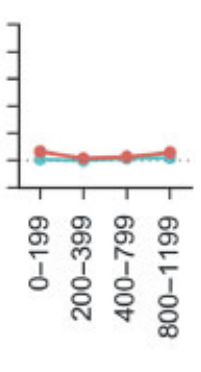

- CD42a labelled

Fig. 7 Effects of platelet inhibitors on the mean platelet movement and contractile component. Measured during thrombus formation on collagen at $400 \mathrm{~s}^{-1}$. Performed in a polydimethylsiloxane (PDMS) flow chamber with 5\% CD42a-labelled platelets (red) and annexin V+ platelets (blue). Time-lapse Z-stack images were captured with wide-field fluorescence microscopy. (A) Illustration of how the velocity component for the contraction $\left(v_{\text {cont }}\right)$ was determined from the movement of individual platelets $v_{x y z}$. (B) Mean total movement velocity $(\mu \mathrm{m} / \mathrm{s})$, (C) length of displacement and the (D) contractile component (\%) over four time stages of the movement. (E) The mean net contractile movement, with and without inhibition, was quantified at different heights throughout the thrombus. ${ }^{* * *} p<0.001,{ }^{*} p<0.05$, mean \pm standard deviation (SD) $(n=6-10)$, the effect of inhibitors was tested as different from zero. Mean $\pm 95 \%$ boot strapped confidence interval $(\mathrm{Cl})(n=6-10)$.

cangrelor or 6B4 that had a remaining significant contractile component.

Although the bulk of the experiments were performed at venous shear rate $\left(400 \mathrm{~s}^{-1}\right)$, an additional set of experiments was performed with increasing shear rates $(133,400,1,200$ and $3,600 \mathrm{~s}^{-1}$ ) to investigate the effects of shear on platelet movements. The platelet count and thrombus height for these experiments are presented in -Supplementary Fig. $\mathbf{5 6}$ (available in the online version) and graphs presenting the velocity, displacement and contraction are presented in - Supplementary Fig. $\mathbf{S 7}$ (available in the online version).

The platelet accumulation was affected by the lowest and highest shear rates. After 600 seconds, there was a significantly lower number of CD42a-labelled platelets in the thrombus at the lowest shear rate, $133 \mathrm{~s}^{-1}(3,650 \pm 670)$ as compared to $400 \mathrm{~s}^{-1}(5,030 \pm 1,100)$. At the highest shear rate $\left(3,600 \mathrm{~s}^{-1}\right)$ platelet accumulation was delayed $(3,860+2,511)$. For the annexin $\mathrm{V}+$ platelets, the accumulation was decreased at the highest shear rate $(460 \pm 244)$ when compared to $400 \mathrm{~s}^{-1}$ (980 \pm 360$)$.

The movement, displacement and contraction were not remarkably affected by the different shear rates (-Supplementary Fig. S7, available in the online version).
There were no significant differences in the mean velocity over the entire experiment independent of shear rate. However, there was significantly less platelet contraction during the experiment performed at 3,600 $\mathrm{s}^{-1}$ compared to $400 \mathrm{~s}^{-1}$ and significantly less contraction of the annexin $\mathrm{V}+$ platelets in the lowest shear rate compared to the $400 \mathrm{~s}^{-1}$. The differences in - Fig. $\mathbf{7}$, at the highest shear rate, are presumably due to the slower initial accumulation in combination with a high degree of embolization of larger platelet aggregates. There were remarkably small differences in the movement between the tested shear rates (- Supplementary Fig. S7A and S7B, available in the online version). However, there are low numbers of platelets at $133 \mathrm{~s}^{-1}$ and initially lower numbers of platelets at $3,600 \mathrm{~s}^{-1}$ (- Supplementary Fig. S6, available in the online version), thus the differences in displacement and contraction could be attributed to either the shear itself or the low platelet number.

\section{Discussion}

In this study, we introduce new computational tools for use in microscopy-based experimental models of thrombosis, enabling quantitative estimations of platelet intra-thrombus 
movements. This approach represents an expansion of our previously published method, where labelling a fraction of the platelets enabled accurate, observer-independent detection and localization of individual platelets within the forming thrombus. ${ }^{18}$ Using an experimental model based on in vitro flow chambers, wherein platelets are activated by immobilized collagen, we applied a tracking algorithm to map the trajectories of platelets inside the thrombus. In this setting, we compare the effects of a panel of platelet inhibitors.

Techniques to track the movements of individual platelets during thrombus formation have been reported previously. ${ }^{11,24}$ However, as these methods only allow for analysis of a limited number of platelets concurrently during one experiment they can only provide information about the movements in the proximity of those few labelled platelets. In addition, previously reported methods are heavily reliant on manual measurements, and are thus characterized by a high degree of observer dependency. In contrast, with our method it is possible to acquire observer-independent information from thousands of platelets dispersed throughout the thrombus, providing a unique and detailed analysis of platelet intra-thrombus movements. Although the thrombi examined in this study were formed on collagen patches of approximately $200 \times 400 \mu \mathrm{m}$, the method is capable of determining the movements of platelets within thrombi of all sizes, the upper limit being determined only by the microscopes' ability to capture the full thrombus structure.

By developing advanced analysis tools for readily available equipment such as wide-field microscopy, we hope that more researchers can have access to more advanced techniques. The versatility and flexibility of this method is therefore a strong advantage as compared to using more specialized tools that does not always fit the research question. However, the method may need to be adapted to each user and therefore requires a user with interest and knowledge in programming.

Two platelet sub-groups were labelled and analysed in all experiments (platelets labelled with a platelet-specific antibody, CD42a and pro-coagulant platelets detected by annexin V). Interestingly, the accumulation of pro-coagulant platelets was only marginally affected by blocking the interactions between the platelet GPs (GPIIb/IIIa, GPIb $\alpha$ ) and their main ligands (fibrinogen, vWF) or by inhibiting thromboxane or P2Y $\mathrm{Y}_{12}$, while the number of CD42a-labelled platelets was dramatically reduced by all treatments, as seen in - Fig. 2 and quantified in - Table 1. As collagen was used as adhesive surface, the contribution of the collagen receptors GPVI and GPIa/IIa to platelet accumulation will also be substantial. However, as collagen is not present within the thrombus itself, the role of the collagen receptors within the thrombus movements could therefore be assumed to be negligible.

These observations support that most clinically used platelet inhibitors preferentially act by reducing the size of the shell in the recently described core-shell model of thrombosis, while leaving the core containing strongly activated platelets relatively intact. ${ }^{7}$ Despite these dramatic differences in inhibitor effects on the accumulation of plate- lets from different platelet sub-populations, all treatments reduced the displacement length and contraction to a similar extent, independent on the platelet sub-group. This result identifies the aggregating non-pro-coagulant platelet as the key driver of thrombus contraction, with the capacity to induce passive contractile movements in pro-coagulant platelets, supposedly by the exertion of contractile mechanical forces on neighbouring cells. In the presence of inhibitors, the drastically reduced contractile forces resulting from the reduction of the number of pro-aggregatory platelets give rise to stochastic platelet movements without direction, as evidenced by the reduced displacement length and contractile movement but preserved overall movements of platelets during these conditions (presented in $\boldsymbol{- F i g . ~} \mathbf{7}$ and $\boldsymbol{-}$ Table $\mathbf{1}$ ). Platelet movements and contraction was also analysed at different shear rates. The shear rate had a relatively small effect on the analysed parameters. However, at the highest shear rate $\left(3,600 \mathrm{~s}^{-1}\right)$ the high degree of embolization hindered a comparative analysis over time.

As can be observed in -Fig. 1, there is some platelet accumulation outside the dotted lines. This is due to single collagen strands outside the coated area. In this article, we analysed all platelets accumulating in the field of view, this also includes the full width of the flow channel, thereby including the areas adjacent to the flow chamber wall where the flow rates are reduced. The objective was to analyse and describe all that was observable in the field of view, thereby illustrating the usability and possibilities of the method. Excluding the platelet within the $10 \%$ of the outermost parts at each side of the channel, to reduce any artefacts or edge effects created by the flow profile of this area, had minor effects on the mean movement (-Supplementary Fig. 55, available in the online version).

The study was performed without coagulation with the focus on developing, evaluating and showing the usability of the presented method. With this scope, we focused on evaluating the platelet movement and platelet contractile forces within the thrombus. This choice was done as allowing coagulation limits the time available for experiments (when corn trypsin inhibitor is used) or affect platelet functionality ${ }^{25}$ (with the addition of citrate) and may also take the focus away from the analysis method. However, in future studies allowing coagulation may be beneficial to give a more physiological picture.

We observed a gradual increase in the contractile component of platelet movements during the first 800 seconds of thrombus formation ( - Fig. 7D). This phenomenon cannot be ascribed to a gradual force generation in individual platelets, as the contractile apparatus has been shown to be rapidly mobilized upon platelet activation. ${ }^{11} \mathrm{~A}$ more plausible explanation can be found when analysing how platelet movements are co-ordinated within the thrombus. As evident from - Fig. 6, platelets initially organize into smaller discrete clusters with separate contractile foci. Over time (after approximately 3 minutes), these contractile microdomains are merged into one single contractile unit spanning thousands of platelets. As our analysis of clot contraction in - Fig. 7D only registers movements directed towards the 
centre of mass, of the entire thrombus, as contractile, the initial movements directed towards different contractile foci are not categorized as contractile using the applied computational model, thereby not estimating that initial contraction. Quantification of these initial heterogeneous contractile movements would require a more sophisticated computational analysis wherein the model would allow for the division of the thrombus into different contractile foci which subsequently would merge into larger clusters, eventually encompassing the entire thrombus.

Building on previous findings, ${ }^{7,8,16}$ our results emphasize the spatial and functional heterogeneity of the forming thrombus, and help to explain how this heterogeneity is developed as a result of platelet intra-thrombus movements. Thrombus movement heterogeneity is evident from the visualizations of movements in -Figs. 4-6, and also when analysing the movement and contraction at different distances from the collagen-coated surface (-Fig. 7E). The increased movement observed higher up in the thrombus structure are partially explained by the absence of any strong adhesive forces anchoring the platelets to the extracellular matrix of the endovasculature (in these experiments represented by the collagen-coated strip at the bottom of the flow chamber). However, a higher degree of movement and contraction can also be observed in the periphery of the thrombus, irrespective of the distance from the collagen-coated surface (-Fig. 4). This phenomenon can partially be attributed to continuous recruitment of new platelets in these areas which are pulled closer to the thrombus surface. However, increased movement in the periphery is still evident at the later stages of thrombus development when the platelet numbers have been stabilized. This is maybe the result of inter-cellular co-operative force generation, as the contractile forces generated by the myosin-actin filaments of a number of individual platelets are connected into functional contractile units via GP receptors anchored to the actin cytoskeleton. How such inter-cellular contractile units spanning a large number of aligned platelets are formed, and how contractions are co-ordinated between cells, is a question outside the scope of this study. Interestingly, recent work has suggested that the formation of gap junctions between platelets mediated by connexin 37 and connexin $40^{26,27}$ could be such a mechanism by which the forces generated by individual platelets are co-ordinated to enable the thrombus to act as a single contractile unit, with the co-operative transmission of contractile forces to the periphery of the thrombus.

The fate of a thrombus is regulated by a number of physiologically important processes such as platelet recruitment, thrombus contraction, microdomain formation and embolization. To adequately map the effects of different conditions such as alterations of blood flow or treatment with drugs, it is necessary to develop models capable of distinguishing these different processes while simultaneously giving quantitative estimates of the net effects on bulk properties such as thrombus mass and shape. The method presented herein for the first time enables such sophisticated thrombus modelling by providing a unique insight into the activities of thousands of platelets dispersed inside the growing thrombus. Although the experiments presented herein are restricted to a simplistic in vitro thrombus model wherein platelets were activated by immobilized collagen and coagulation was inhibited, our tracking method could readily be transferred to more comprehensive experimental models and extended to incorporate additional analysis features. The tracking method is suitable for in vitro systems with other activating surfaces, both proteins and cells provided it is possible to acquire adequate microscopy images. The method could also be adapted for intra-vital microscopy to study thrombosis in vivo. Such models could help to answer several important questions such as how platelet movements contribute to the formation of the recently described core-shell thrombus structure.

Herein, we have shown that it is possible to track and discriminate between CD42a-labelled and annexin V+ platelets. However, the method could also be adapted to distinguish other platelet sub-populations based on fluorescent markers or differences in size. Admittedly, studies on differences in shape would necessitate improved image resolution.

\section{What is known about this topic?}

- With the build-up of a platelet plug at a site of vascular injury, platelet recruitment occurs in parallel with thrombus densification due to the generation of platelet contractile forces, causing simultaneous compaction and expansion of the thrombus.

- A more complete understanding of thrombosis would require experimental methods capable of mapping the activities and movements of a large number of individual platelets inside the thrombus.

\section{What does this paper add?}

- New computational tools for use in microscopy-based experimental models of thrombosis, enabling quantitative estimations of platelet intra-thrombus movements.

- Thrombus contraction was quantified and we observed a gradual increase in the contraction during the first 800 seconds of thrombus formation.

Authors' Contributions

$K$. Tunströmer performed the experiments and wrote the first draft of the manuscript. K. Tunströmer and L. Faxälv performed data analysis. All authors contributed to the design of the study, interpretation of results, writing and final approval of the manuscript.

\section{Funding}

This study was supported by a grant from the Swedish Research Council VR3R, Project No K2015-79X-22644-01-3 
and DNr 2017-01177, the Swedish Heart-Lung foundation No 2017-0440 and by Linköping University.

\section{Conflict of Interest}

None.

\section{References}

1 de Witt SM, Swieringa F, Cavill R, et al. Identification of platelet function defects by multi-parameter assessment of thrombus formation. Nat Commun 2014;5(May):4257

2 Nieswandt B, Brakebusch C, Bergmeier W, et al. Glycoprotein VI but not alpha2beta1 integrin is essential for platelet interaction with collagen. EMBO J 2001;20(09):2120-2130

3 Maloney SF, Brass LF, Diamond SL. P2Y12 or P2Y1 inhibitors reduce platelet deposition in a microfluidic model of thrombosis while apyrase lacks efficacy under flow conditions. Integr Biol 2010;2(04):183-192

$4 \mathrm{Li}$, Diamond SL. Detection of platelet sensitivity to inhibitors of COX-1, $\mathrm{P}_{2} \mathrm{Y}_{1}$, and $\mathrm{P}_{2} \mathrm{Y}_{12}$ using a whole blood microfluidic flow assay. Thromb Res 2014;133(02):203-210

5 Lee H, Sturgeon SA, Jackson SP, Hamilton JR. The contribution of thrombin-induced platelet activation to thrombus growth is diminished under pathological blood shear conditions. Thromb Haemost 2012;107(02):328-337

6 Tovar-Lopez FJ, Rosengarten G, Westein E, et al. A microfluidics device to monitor platelet aggregation dynamics in response to strain rate micro-gradients in flowing blood. Lab Chip 2010;10 (03):291-302

7 Stalker TJ, Traxler EA, Wu J, et al. Hierarchical organization in the hemostatic response and its relationship to the platelet-signaling network. Blood 2013;121(10):1875-1885

8 Munnix ICA, Kuijpers MJE, Auger J, et al. Segregation of platelet aggregatory and procoagulant microdomains in thrombus formation: regulation by transient integrin activation. Arterioscler Thromb Vasc Biol 2007;27(11):2484-2490

9 Heemskerk JWM, Mattheij NJA, Cosemans JMEM. Platelet-based coagulation: different populations, different functions. J Thromb Haemost 2013;11(01):2-16

10 Tutwiler V, Litvinov RI, Lozhkin AP, et al. Kinetics and mechanics of clot contraction are governed by the molecular and cellular composition of the blood. Blood 2016;127(01):149-159

11 Ono A, Westein E, Hsiao S, et al. Identification of a fibrinindependent platelet contractile mechanism regulating primary hemostasis and thrombus growth. Blood 2008;112(01):90-99

12 Léon C, Eckly A, Hechler B, et al. Megakaryocyte-restricted MYH9 inactivation dramatically affects hemostasis while preserving platelet aggregation and secretion. Blood 2007;110(09):3183-3191
13 Lam WA, Chaudhuri O, Crow A, et al. Mechanics and contraction dynamics of single platelets and implications for clot stiffening. Nat Mater 2011;10(01):61-66

14 Aoki N. Clot retraction increases clot resistance to fibrinolysis by condensing alpha 2-plasmin inhibitor crosslinked to fibrin. Thromb Haemost 1993;70(02):376

15 Sutton JT, Ivancevich NM, Perrin SRJ Jr, Vela DC, Holland CK. Clot retraction affects the extent of ultrasound-enhanced thrombolysis in an ex vivo porcine thrombosis model. Ultrasound Med Biol 2013;39(05):813-824

16 Munnix ICA, Cosemans JMEM, Auger JM, Heemskerk JWM. Platelet response heterogeneity in thrombus formation. Thromb Haemost 2009;102(06):1149-1156

17 Welsh JD, Stalker TJ, Voronov R, et al. A systems approach to hemostasis: 1 . The interdependence of thrombus architecture and agonist movements in the gaps between platelets. Blood 2014;124(11):1808-1815

18 Claesson K, Lindahl TL, Faxälv L. Counting the platelets: a robust and sensitive quantification method for thrombus formation. Thromb Haemost 2016;115(06):1178-1190

19 Fontayne A, Vanhoorelbeke K, Pareyn I, et al. Rational humanization of the powerful antithrombotic anti-GPIbalpha antibody: 6B4. Thromb Haemost 2006;96(05):671-684

20 Roest M, Reininger A, Zwaginga JJ, King MR, Heemskerk JWM; Biorheology Subcommittee of the SSC of the ISTH. Flow chamberbased assays to measure thrombus formation in vitro: requirements for standardization. J Thromb Haemost 2011;9(11): 2322-2324

21 Van Kruchten R, Cosemans JMEM, Heemskerk JWM. Measurement of whole blood thrombus formation using parallel-plate flow chambers - a practical guide. Platelets 2012;23(03):229-242

22 Heemskerk JW, Vuist WM, Feijge MA, Reutelingsperger CP, Lindhout T. Collagen but not fibrinogen surfaces induce bleb formation, exposure of phosphatidylserine, and procoagulant activity of adherent platelets: evidence for regulation by protein tyrosine kinase-dependent $\mathrm{Ca} 2+$ responses. Blood 1997;90(07): 2615-2625

23 Crocker J, Grier D. Methods of digital video microscopy for colloidal studies. J Colloid Interface Sci 1996;179(01):298-310

24 Stalker TJ, Welsh JD, Tomaiuolo M, et al. A systems approach to hemostasis: 3 . Thrombus consolidation regulates intrathrombus solute transport and local thrombin activity. Blood 2014;124(11): 1824-1831

25 Neeves KB, Onasoga AA, Hansen RR, et al. Sources of variability in platelet accumulation on type 1 fibrillar collagen in microfluidic flow assays. PLoS One 2013;8(01):e54680

26 Vaiyapuri S, Jones CI, Sasikumar P, et al. Gap junctions and connexin hemichannels underpin hemostasis and thrombosis. Circulation 2012;125(20):2479-2491abstract

27 Vaiyapuri S, Moraes LA, Sage T, et al. Connexin40 regulates platelet function. Nat Commun 2013;4:2564 